\title{
Requirements Smells como indicadores de má qualidade na especificação de requisitos: Um Mapeamento Sistemático da Literatura
}

\author{
Rafael Nascimento, Eduardo Aranha, Uirá Kulesza, e Marcia Lucena \\ Departamento de Informática e Matemática Aplicada, Universidade Federal do Rio \\ Grande do Norte, Natal, Brasil \\ \{rafael.jullian, eduardohsaranha, uirakulesza,marcia.lucena\}@gmail.com
}

\begin{abstract}
Resumo Artefatos de requisitos são primordiais para guiar as demais etapas do processo de desenvolvimento e garantir a qualidade do produto. Quando os artefatos são de má qualidade, estes sintomas tendem a ser propagados para as demais etapas, ocasionando mais problemas de qualidade. Diante disso, pesquisadores têm investigado a existência de indicadores de má qualidade na especificação de requisitos, prejudicando negativamente a compreensão, manutenção e qualidade dos artefatos. Estes indicadores são descritos como Requirements Smells e são utilizados para inspecionar a qualidade das especificações de requisitos em linguagem natural. Este trabalho apresenta um Mapeamento Sistemático da Literatura para compreender como o tema está sendo abordado, seu conceito, o nível dos suportes ferramentais e análise em projetos reais. Um total de 41 estudos foram selecionados e que desde 2013, trabalhos vêm sendo produzidos de forma crescente. Compreende-se que o conceito tem ganhado visibilidade, existência de suportes ferramentais, aplicação do conceito de Requirements Smells na inspeção de requisitos em projetos reais e o seu impacto em outras etapas do desenvolvimento de software.
\end{abstract}

Palavras-Chaves: Engenharia de Requisitos · Análise de Qualidade · Requirements Smells.

\section{Introdução}

A etapa de Engenharia de Requisitos é essencial para o processo de desenvolvimento de software; pois os artefatos de requisitos são importantes para o planejamento arquitetural, implementação das funcionalidades e planejamento de testes para verificação e validação do sistema [18]. Porém, especificar requisitos de qualidade não é uma tarefa trivial, devido as diversas necessidades dos stakeholders, existência de subjetividade na compreensão e interpretação dos requisitos; podendo comprometer a sua qualidade, devido requisitos implementados de forma equivocada até requisitos que não foram solicitados pelo cliente [46]. Caso os artefatos de requisitos sejam de má qualidade, isto pode comprometer o desenvolvimento e a qualidade do produto final [18]. Diante disso, normas foram 
criadas para ajudar na qualidade das especificações, como a norma IEEE 830 [1] e ISO 29148 [2].

Apesar das normas existentes, alguns pesquisadores notaram a existência de sintomas de má qualidade na especificação de requisitos em linguagem natural, e que estes tendem a causar impacto negativo na qualidade dos documentos e em demais atividades deles dependentes; estes sintomas são chamados de Requirements Smells [18]. Requirements Smells são caracterizados como indicadores de má qualidade na especificação de requisitos em linguagem natural. Além disso, pesquisas estão sendo desenvolvidas na área utilizando Requirements Smells em metodologias, suportes ferramentais e na prática com sistemas industriais para melhoria da qualidade dos processos de especificação.

Todavia, até o momento, nenhum Mapeamento Sistemático desta Literatura foi realizado para uma melhor compreensão da área e sobre quais tipos de pesquisas estão sendo realizadas. O objetivo deste trabalho consiste em um Mapeamento Sistemático da Literatura sobre o tema de Requirements Smells, para ajudar na compreensão do tema e auxiliar pesquisadores e praticantes em estudos futuros.

Este trabalho está organizado da seguinte forma: na Seção 2, é abordado a estrutura do Mapeamento Sistemático da Literatura realizado; na Seção 3, são apresentados os trabalhos selecionados, os resultados e suas discussões; na Seção 5, são descritos alguns trabalhos relacionados a esta pesquisa; na Seção 4, as limitações deste estudo e na Seção 6, as considerações finais deste mapeamento sistemático.

\section{Método de Pesquisa}

Um Mapeamento Sistemático da Literatura (MSL) tem por finalidade classificar e representar uma área de pesquisa, descrevendo tópicos que estão sendo abordados para estudo [10]. A principal meta desta MSL não foi selecionar todas as evidências de estudo, mas fornecer orientações para praticantes e pesquisadores sobre quais tipos de estudos estão sendo desenvolvidos sobre o tema abordado. O MSL realizado seguiu a metodologia proposta no trabalho de [35].

\subsection{Questões de Pesquisa}

Foram definidas 4 questões de pesquisas para guiar o processo de busca e mapeamento dos estudos. Os objetivos consistem em encontrar evidências de estudo, sobre a definição do tema, propostas de suporte ferramental e aplicações práticas na academia ou industriais:

QP1: Qual definição está sendo utilizada para Requirements Smells? Tem por objetivo verificar se os pesquisadores estão em consenso com uma determinada definição ou se várias definições estão sendo utilizadas.

QP2: Existe catálogo sobre Requirements Smells sendo utilizado? Tem por objetivo verificar se existe um ou mais catálogos que podem ser aplicados para identificação de Requirements Smells. 
QP3: São propostos métodos ou ferramentas para detecção de Requirements Smells? Tem por finalidade verificar se existe suporte ferramental para auxiliar na detecção automática e/ou semi-automática de Requirements Smells.

QP4: Como Requirements Smells está sendo aplicado na prática? Tem por objetivo verificar se as pesquisas sobre Requirements Smells vem sendo aplicados na área de Engenharia de Requisitos e/ou demais áreas da Engenharia de Software.

\subsection{Processo de Busca}

O processo de busca de estudos foi realizada de forma automatizada, recuperando pesquisas com data de publicação de Janeiro de 2013 até Março de 2018. A justificativa para a escolha desta data inicial é devido o primeiro estudo, utilizando o termo Requirements Smells de forma sistematizada e definida no contexto da Engenharia de Requisitos, ter sido publicado nesta data (de acordo com o resultado de uma busca piloto). Não foi objetivo deste estudo, realizar uma busca Snownballing para encontrar literaturas utilizando nomes semelhantes. A busca foi realizada em cinco bibliotecas digitais, sendo elas: Google Scholar, IEEE Xplore, ACM Digital Library, Science Direct e Digital Bibliography $\&$ Library Project(DBLP). Foi construída e utilizada a seguinte string de busca:

\footnotetext{
"requirement smell" OR "requirements smells" OR "requirement anomaly" OR "requirements anomalies"
}

A justificativa para a estrutura da nossa string de busca, se deve ao fato de termos semelhantes consolidados como Code Smell, Codes Anomalies Architectural Smell e Architectural Anomaly, estarem sendo usados em campos da Arquitetura e Linha de Produto de Software [30,48]. Optou-se não utilizar o termo Bad Smell por ser um termo muito genérico, cuja busca seria muito ampla e exaustiva trazendo resultados que fogem do escopo desta pesquisa.

\subsection{Critérios de Inclusão e Exclusão}

Para filtrar e eliminar publicações irrelevantes e fora do escopo de nossas questões de pesquisa, foram adotados os seguintes critérios de inclusão e exclusão. Foram incluídos artigos científicos que descrevem definições, suporte ferramental, aplicação prática para a qualidade dos requisitos ou em outras áreas; e trabalhos que fazem referência ao termo ou algum tipo de Requirement Smell. Foram excluídas publicações que estão fora do escopo de estudo, publicações que não são científicas, teses, dissertações e capítulos de livros.

\section{Resultados}

\subsection{Resultados da busca}

A filtragem dos estudos, de acordo com os critérios de exclusão, aconteceu em quatro etapas: identificação de 118 estudos relevantes utilizando os itens definidos na busca (Filtro 1); exclusão de 43 estudos, filtrando pelo título, resumo, 
introdução e palavras-chaves (Filtro 2); exclusão de 16 estudos, pela leitura completa do artigo (Filtro 3); exclusão de 18 estudos duplicados (Filtro 4). No total, 41 trabalhos foram selecionados neste estudo. A Tabela 1 ilustra a quantidade de trabalhos publicados por ano. Em todos os anos, de 2013 à 2018, estudos vêm sendo publicados de forma crescente e a maior quantidade de publicações ocorre em 2017 (19 publicações). A quantidade de trabalhos publicados em 2017, pode indicar que o tema vem ganhando visibilidade e sendo fonte de investigação na comunidade acadêmica.

Tabela 1. Classificação das publicações por ano.

\begin{tabular}{lllllllll}
\hline & \multicolumn{3}{c}{ Ano } & & & & \multicolumn{2}{c}{ Total } \\
\cline { 2 - 7 } & 2013 & 2014 & 2015 & 2016 & 2017 & Até & Março 2018 & \\
\hline Publicações & 1 & 3 & 4 & 7 & 19 & 7 & 41 \\
\hline
\end{tabular}

\subsection{Discussão dos Resultados}

QP1: Qual definição está sendo utilizada para Requirements Smells? No trabalho de [18] foi proposto o termo Requirement Smell como indicador de sintomas de má qualidade na especificação de requisitos em linguagem natural em um contexto de uso de um determinada atividade; baseado nos padrões de qualidade das normas IEEE 830 [1] e ISO 29148 [2]. No trabalho de [17] foi descrito como indicador na busca por violações em critérios comuns de qualidade, baseada no processamento de linguagem natural. No trabalho de [20], baseado na ISO 29148, foi descrito que são sintomas de defeitos na qualidade de requisitos. Nos trabalhos de $[7,41]$ foi descrito que são sinais de deficiência em requisitos e um concreto sintoma para defeito de qualidade. Nos trabalhos $[5,12]$ foi descrito que podem ser sinais de imprecisão ou ambiguidade na declaração de requisitos, causando alto custo de refatoração. Nos trabalhos de [7,8,21], Requirements Smells foi definido de forma mais detalhada e contextualizada, onde estas anomalias são caracterizadas por:

1. Violação de qualidade em um artefato de requisitos em termos de uso, onde o artefato de requisitos de má qualidade afeta negativamente atividades do ciclo de vida de software dependentes destes artefatos;

2. Não necessariamente leva a um defeito em um determinado contexto, pois depende das decisões individuais, revisões e outras atividades para garantia de qualidade;

3. A anomalia possui uma localização concreta em uma entidade presente em um artefato de requisitos;

4. A anomalia tem um mecanismo de detecção concreto;

5. Pode prejudicar a legibilidade do documento de requisitos, podendo levar ao mal entendimento entre os autores e leitores dos documentos. 
Foi observado que existe um consenso sobre a definição do termo Requirement Smell entre os diversos estudos e que de um modo geral, são considerados como indicadores de má qualidade na especificação de requisitos em linguagem natural. Impactando negativamente a qualidade dos artefatos e das demais atividades de desenvolvimento. No mais, os trabalhos de $[9,44]$ somente citam a existência de Requirements Smells como um conceito investigado por [21].

QP2: Existe catálogo sobre Requirements Smells sendo utilizado? Nesta pesquisa, somente dois trabalhos achados descrevem um mesmo catálogo [20,21]. Demais trabalhos aplicam ou descrevem alguns tipos de Requirements Smells. Alguns trabalhos utilizam nomenclaturas ou descrevem anomalias diferentes do catálogo esposto [20]. Os tipos de Requirements Smells descritos por [20,21], são:

- Ambiguous Adverbs and Adjectives: são adjetivos e advérbios que causam ambiguidade na compreensão dos requisitos. Exemplo: Se a qualidade (...) for muito baixa, uma falha deve ser gravada na memória de erros.

- Vague Pronouns: são pronomes com relações pouco claras. Exemplo: O software deve implementar serviços para aplicativos, que devem se comunicar com os aplicativos do controlador implantados em outros controladores.

- Subjective Language: são palavras cuja semântica não é objetiva. Exemplos: amigável, fácil de usar, econômico.

- Comparative: são advérbios e adjetivos, onde os requisitos expressam uma relação do sistema com outros sistemas específicos. Exemplo: melhor que, maior qualidade.

- Superlative: são advérbios e adjetivos, onde os requisitos expressam uma relação do sistema com todos os outros sistemas. Exemplo: melhor desempenho, menor tempo de resposta.

- Negative Statements: são palavras usadas em funcionalidades que o sistema não deve fornecer, pois podem levar a falta de explicação sobre o comportamento do sistema em tais casos. Exemplo: o sistema não deve aceitar cartões de crédito VISA.

- Non-verifiable terms: são palavras difíceis de verificar por oferecer várias possibilidades de execução do sistema. Exemplo: O sistema só pode ser ativado se todos os sensores necessários (...) trabalharem com precisão de medição suficiente.

- Loopholes: são palavras que possibilitam os stakeholders ignorar as especificações. Exemplos: se possível, conforme apropriado, conforme aplicável.

- Incomplete references: São referências que o leitores não conseguem encontrar;

No trabalho de [41] são citadas as anomalias Ambiguous Adverbs and Adjectives, Vague Pronouns, Negative Statements e Subjective Language. Também foi descrita uma anomalia chamada Duplicate Funcionality [11], caracterizada pela ação de repetir descrições de mesmas interações entre sistemas e atores em várias especificações de casos de uso. No trabalho de [38] foi realizada uma investigação na anomalia Negative Statements sobre o seu impacto no teste de sistema em 
termos de omissões ou casos de testes incorretos. No trabalho de [49] foi descrita um outro tipo de anomalia Passive Voice, caracterizada por requisitos onde não está claro o ator que está desempenhando uma determinada ação no sistema.

No trabalho de [3] foi introduzido 16 padrões para testar a qualidade de ferramentas para análise de requisitos especificados em linguagem natural, considerando somente violações de ambiguidade e atomicidade. Entre estes padrões, estão: And, Or, Multiple Features, List of items, Multiple sentences, Quantifier, Unit, Slash, General Ambiguity e Escape Clause. E também são incluídos alguns padrões mencionados em [20] como Requirements Smells, são: Vague Pronouns, Non-verifiable Terms, Subjective Terms, Comparative Terms, Negative Terms e Superlative Terms.

No trabalho de [50] foi realizada uma análise de ambiguidade em requisitos, fazendo referência a anomalias como Subjective Terms e Non-Verifiable Terms. No trabalho de [47] foi referenciada a anomalia Ambiguous Adverbs e no trabalho de [8] foram referenciadas as anomalias Passive Voice, Imprecise Phrase (catalogada por [20,21] como Loopholes) e Vague Pronouns.

Nos trabalhos de $[28,43]$ são descritas classes para detecção de defeitos em requisitos. Alguns destes defeitos são chamados de Requirements Smells nos trabalhos de [20,21,49]: Ambiguity Adverbs como Modal Adverbs, Incomplete Reference como Missing Reference, Non-verifiable Terms como Coordination Ambiguity, Passive Voice, Vague Pronouns como Anaphoric Ambiguity e Vague Terms.

Observa-se que algumas pesquisas estão investigando problemas de má qualidade na especificação de requisitos e fazendo uso do catálogo de Requirements Smells proposto por [20] e apresentado em [21]. Também, outras anomalias que não estão presentes no catálogo proposto por [20] estão sendo investigadas. Na Tabela 2, são descritos as anomalias organizadas por trabalhos. De um modo geral, a maioria dos estudos investigam problemas relacionados a ambiguidade, termos não compreensíveis e sentenças negativas.

Tabela 2. Requirements Smells por estudos selecionados.

\begin{tabular}{lll}
\hline Requirements Smells & Estudo(s) & Total \\
\hline Ambiguous Adverbs and Adjectives & {$[20,21,28,41,43,47]$} & 6 \\
\hline Vague Pronouns & {$[3,8,20,21,28,41,43]$} & 7 \\
\hline Subjective Language & {$[3,20,21,41,50]$} & 5 \\
\hline Comparative & {$[3,20,21]$} & 3 \\
\hline Superlative & {$[3,20,21]$} & 3 \\
\hline Negative Statements & {$[3,20,21,38,41]$} & 5 \\
\hline Non-verifiable terms & {$[3,20,21,28,43,50]$} & 6 \\
\hline Loopholes & {$[8,20,21]$} & 3 \\
\hline Incomplete References & {$[20,21,28,43]$} & 4 \\
\hline Duplicate Funcionality & {$[11]$} & 1 \\
\hline Passive Voice & {$[8,28,43,49]$} & 4 \\
\hline
\end{tabular}


QP3: São propostos métodos ou ferramentas para detecção de Requirements Smells? Foi verificado que suportes ferramentais estão sendo propostos para detecção automatizada de Requirements Smells. As metodologias propostas visam melhorar a qualidade dos processos de especificação de requisitos, onde alguns trabalhos sugerem incorporar Requirements Smells como indicadores de má qualidade. Além disso, foram encontrados trabalhos que fazem referências as ferramentas utilizadas para detecção de Requirements Smells propostas na literatura.

A Tabela 3 ilustra as ferramentas organizadas por trabalhos que propõem, trabalhos que mencionam e trabalhos que utilizam ferramentas de terceiros. A maioria dos trabalhos citam ferramentas de terceiros para deteç̧ão de $R e$ quirements Smells, relacionados à problemas de ambiguidade e inconsistência, conforme o padrão ISO 29148 para a qualidade de requisitos, utilizando PLN. Somente dois trabalhos utilizam ferramentas propostas por terceiros.

Tabela 3. Ferramentais e/ou metodologias por estudos selecionados.

\begin{tabular}{llc}
\hline & & Total \\
\hline Propõem ferramentas & {$[5,7,20,21,43,50]$} & 6 \\
\hline Propõem metodologias & {$[14,16,23,24]$} & 4 \\
\hline Citam ferramentas de terceiros & {$[4,15,19,22,25,26,27,28,29,33,36,41,43,45,47,50,51]$} & 17 \\
\hline Utilizam ferramentas de terceiros & {$[3,8]$} & 2 \\
\hline
\end{tabular}

No trabalho de [20] foi proposta uma ferramenta para deteç̧ão automática de Requirements Smells, utilizando técnica de Processamento de Linguagem Natural (PLN), verificando requisitos ou casos de uso de forma individual. No trabalho de [5] foi proposta uma ferramenta para verificar se as especificações de requisitos, estão em conformidade com os templates Rupp [40] ou EARS [37]. Os autores mencionam que a sua ferramenta é capaz de detectar e emitir alertas sobre Requirements Smells descritos em [20].

No trabalho de [21] foi proposta a ferramenta protótipo, chamada SMELLA, para detecção de Requirements Smells. De forma semelhante, no trabalho de [50] foi proposta uma ferramenta chamada Tactile Check, para detectar ambiguidade em documentos de requisitos baseado no catálogo em [20]. Em [43], os autores fizeram a inclusão de técnicas de PLN em uma ferramenta para processamento de textos, chamada GATE [13]. A ferramenta foi utilizada para detectar problemas de qualidade em documentos de requisitos como: Vague Terms, Passive Voice, Missing Reference, Modal Adverbs, Anaphoric Ambiguity, Non-verifiable Terms e Coordination Ambiguity. Em [7] foi proposto um protótipo ferramental, onde o usuário especifica requisitos em linguagem natural e através de PLN, é realizada uma análise para detectar erros e pontos fracos relacionados à ambiguidade e incompletude nos requisitos baseado em [20].

Nos trabalhos [16,23,24] são descritas as metodologias ABRE-QM (ActivityBased Requirements Engineering Quality Model) para definir a qualidade de artefatos no processo de Engenharia de Requisitos. Os autores sugerem o uso 
de Requirements Smells para fornecer feedback, como indicadores de má qualidade nas especificações. No trabalho de [14] foi apresentada uma metodologia para especificar requisitos de qualidade por meio de atributos de qualidade baseado em padrões. Os autores descrevem que a metodologia pode ser utilizada para fornecer feedback sobre elementos dos requisitos por meio de Requirements Smells.

Ferramentas estão sendo desenvolvidas para detectar Requirements Smells utilizando PLN, devido estas anomalias serem indicadores de má qualidade na especificação de requisitos em linguagem natural. Três ferramentas são propostas para detecção de anomalias apresentadas em [20] e duas para anomalias relacionadas a ambiguidade. As metodologias propostas são para melhoria da qualidade do processo na Engenharia de Requisitos, utilizando Requirements Smells para obter feedback na qualidade dos requisitos especificados.

QP4: Como Requirements Smells está sendo aplicado na prática? Nos trabalhos selecionados, foi observado que a definição e suporte ferramental, relacionados a Requirements Smells, vem sendo aplicado na prática em análise de especificações de requisitos de projetos reais na indústria.

No trabalho de [20] foram realizados dois estudos de caso. Um sistemas de informação de indústria química contendo 303 requisitos e um sistema de engenharia de controle e tráfego, contendo 53 casos de uso e 13 requisitos sem uso de templates. Em [5] foi realizado 4 estudos de caso: um software de uma estação de satélite com 380 requisitos, utilizando os templates Rupp e EARS; uma ferramenta para gerenciamento de segurança da informações com 110 requisitos em template Rupp; um projeto de requisitos de segurança para uma instalação nuclear com 890 requisitos em template EARS.

Em [23] foi inspecionado um sistema de software para gerenciamento de negócios customizado, com 51 requisitos incluindo casos de uso e regras de negócios. Em [21] foi analisado 4 estudos de caso, sendo três estudos de caso industriais e um estudo de caso acadêmico: um sistema de engenharia de controle e automóvel com 323 requisitos; uma ferramentas de gestão de negócios contendo 53 casos de uso e 13 requisitos não numerados; 1000 histórias do usuário de 4 sistemas ágeis sendo dois portais de compras online, um sistema de relacionamento com o cliente e um sistema de gerenciamento de conteúdo; 51 artefatos de requisitos com cerca de 950 casos de uso criado por estudantes.

No trabalho de [3] foi realizada uma análise do desempenho de ferramentas propostas em $[20,34,31]$ que utilizam técnicas de processamento de linguagem natural, que utilizam Requirements Smells. Estas foram analisadas com conjuntos de dados desenvolvidos pelos proprietários das ferramentas com mais um conjunto de user stories desenvolvido pelos autores do trabalho. No trabalho de [50] foram inspecionados documentos com 293 requisitos, de projetos relacionados a Business Intelligence: o e-EGS, uma solução computacional de cargas para suporte ao Boeing 787 escrito em linguagem natural; o CMS-Plus, uma solução logística para gerenciamento da administração e execução de vôos, escrito em linguagem natural como user stories ou casos de uso. 
No trabalho de [47], o estudo de caso foi realizado com Swedish Transport Administration, uma agência governamental responsável pela infraestrutura ferroviária, marítima e da aviação da Suíça. No trabalho não fica claro a quantidade de requisitos analisada, só descrevem que foram milhares. No trabalho de [8] foi analizada uma aplicação web com 37 requisitos. Na documentação de requisitos foram injetadas anomalias como: Passive Voice, Loophole, Negative Words, UI details, Imprecise Phrases, Vague Pronouns. Os autores chegaram a conclusão que Requirements Smells influencia a qualidade dos casos de testes e a identificação destas anomalias podem melhorar o projeto dos casos de teste.

Em [43], foi realizado um estudo empírico na deteç̧ão de anomalias em documentos de requisitos no domínio ferroviário. O sistema Automatic Train Protection com 241 requisitos. O outro estudo foi realizado em um conjunto de 1866 requisitos de um sistema composto por 4 subsistemas.

Tabela 4. Tipos de projetos por estudos selecionados.

\begin{tabular}{lll}
\hline Tipos de projetos & Estudo(s) & Total \\
\hline Projeto(s) industrial(is) $[20,5,23,21,50,47,8,43]$ & 14 \\
\hline Projeto(s) acadêmico(s) [3] & 5 \\
\hline Total de projetos & $\mathbf{1 9}$ \\
\hline
\end{tabular}

Requirements Smells está sendo aplicado para detectar sintomas de má qualidade na especificação de requisitos em sistemas do mundo real e de características diversas (Tabela 4). Também está sendo aplicado em pesquisas envolvendo outras áreas da Engenharia de Software, como a área de Teste de Software. Isso pode indicar que o tema sobre Requirements Smells está sendo consolidado e popularizado.

\section{Limitacões do Trabalho}

Algumas limitações no desenvolvimento deste estudo puderam ser identificadas e são discutidas a seguir. A string de busca utilizada pode ter suprimido trabalhos descrevendo anomalias sobre requisitos utilizando outros termos e/ou definições. A busca pode ter suprimido publicações sobre o assunto em outros idiomas. A faixa de ano utilizada pode ter excluído trabalhos anteriores à 2013, utilizando os termos aplicados nesta pesquisa que não puderam ser achados ou outros termos; é importante frisar que a string de busca foi aplicada em uma busca piloto com data anterior a data de 2013 e não foram encontradas publicações utilizando o termo Requirements Smells. Em algumas bibliotecas digitais foi necessário adaptar a string de busca, por não ser compatível com a regra de busca utilizada neste trabalho. Os procedimentos de filtragem podem ter suprimido publicações abordando o tema, utilizando outros termos. Desse modo, não podemos garantir que todas as publicações relacionadas com o tema puderam ter sido achadas. 


\section{Trabalhos Relacionados}

Outros estudos também foram realizados por meio de revisão ou mapeamento sistemático da literatura para delimitar escopo de trabalhos relacionados a melhoria da qualidade dos artefatos de especificações de requisitos.

No trabalho de [6] foi realizado um MSL para verificar o estado atual de pesquisas na melhoria de documentos de requisitos, suporte ferramental e validações para estas melhorias. Após a análise dos estudos, os autores propõem melhorias e suporte ferramental para a abordagem AIRDoc (Approach to Improve Requirements Documents)[42].

No trabalho de [32] foi realizado uma Revisão Sistemática da Literatura (RSL) para investigar quais critérios de qualidade estão sendo garantidos para a escrita de requisitos ágeis. Os autores categorizaram e classificaram 28 critérios de qualidade, como forma de recomendação aos praticantes, para a especificação de requisitos ágeis.

O trabalho de [39] investigou, por meio de RSL, técnicas para elicitação de requisitos; verificando o seu atual nível de maturidade e se realmente melhoram a qualidade das elicitações. Os autores classificam quais técnicas são mais eficazes e em quais situações são melhores para elicitação, levando em consideração caraterísticas de produtos, stakeholders e entre outras informações.

\section{Considerações Finais}

O presente trabalho apresentou uma Mapeamento Sistemático da Literatura sobre o tema Requirements Smells, para investigar quais estudos vem sendo realizados nesta área e como tem sido validado. Observa-se que desde 2013 até o presente momento o tema tem sido objeto de investigação no meio acadêmico e que, provavelmente, a partir de 2017 o tema vem ganhado popularidade seja como objeto de estudo seja como referências para outros estudos. Considerase que existe um consenso, entre as pesquisas selecionadas, sobre o conceito de Requirements Smells e o seu impacto negativo na qualidade dos artefatos de requisitos e de atividades dependentes. Um catálogo tem sido proposto para auxiliar os pesquisadores na detecção concreta destes sintomas de má qualidade nas especificações; além disso, alguns trabalhos descrevem outros tipos de anomalias. Suportes ferramentais tem sido desenvolvidos e validados pela análise de especificações de requisitos de diferentes sistemas industriais dos mas diversos tamanhos, domínios e paradigmas de desenvolvimento; sendo utilizadas para investigação de inconsistência e ambiguidade em requisitos. No mais, o tema tem sido objeto de investigação sobre o impacto negativo de artefatos de requisitos, infectados por Requirements Smells, no planejamento de casos de testes; indicando que o tema esta sendo investigado com a área de Teste de Software. Logo, observa-se que o tema vem sendo objeto de estudo para metodologias, suportes ferramentais e validações na inspeção de artefatos de requisitos de sistemas industriais. Finalmente, isto pode indicar que um novo tipo de indicador de qualidade para especificação de requisitos esta sendo consolidado, melhorando o processo de Engenharia de Requisitos e demais áreas por ela dependentes. 


\section{Referências}

1. IEEE Recommended Practice for Software Requirements Specifications. IEEE Std 830-1998 pp. 1-40 (1998). https://doi.org/10.1109/IEEESTD.1998.88286

2. Iso/iec/ieee international standard - systems and software engineering - life cycle processes -requirements engineering. ISO/IEC/IEEE 29148:2011(E) pp. 1-94 (Dec 2011). https://doi.org/10.1109/IEEESTD.2011.6146379

3. Arendse, B., Lucassen, G.: Toward Tool Mashups: Comparing and Combining NLP RE Tools. In: 2016 IEEE 24th International Requirements Engineering Conference Workshops (REW). pp. 26-31 (2016). https://doi.org/10.1109/REW.2016.019

4. Arora, C., Sabetzadeh, M., Briand, L., Zimmer, F.: Automated Extraction and Clustering of Requirements Glossary Terms. IEEE Transactions on Software Engineering 43(10), 918-945 (2017). https://doi.org/10.1109/TSE.2016.2635134

5. Arora, C., Sabetzadeh, M., Briand, L., Zimmer, F.: Automated checking of conformance to requirements templates using natural language processing. IEEE transactions on Software Engineering 41(10), 944-968 (2015)

6. Azevedo, R., Sousa, A., Ramos, R., Alencar, F.: Melhoria de documentos de requisitos: um mapeamento sistemático da literatura. Workshop on Requirements Engineering (2016)

7. Bäumer, F.S., Geierhos, M.: Flexible ambiguity resolution and incompleteness detection in requirements descriptions via an indicator-based configuration of text analysis pipelines. In: Proceedings of the 51st Hawaii International Conference on System Sciences (2018)

8. Beer, A., Junker, M., Femmer, H., Felderer, M.: Initial Investigations on the Influence of Requirement Smells on Test-Case Design. In: 2017 IEEE 25th International Requirements Engineering Conference Workshops (REW). pp. 323-326. IEEE (2017)

9. Berry, D.M., Ferrari, A., Gnesi, S.: Assessing tools for defect detection in natural language requirements: Recall vs precision (2018)

10. Budgen, D., Turner, M., Brereton, P., Kitchenham, B.: Using mapping studies in software engineering. In: Proceedings of PPIG. vol. 8, pp. 195-204. Lancaster University (2008)

11. Ciemniewska, A., Jurkiewicz, J., Olek, Ł., Nawrocki, J.: Supporting use-case reviews. In: Proceedings of the 10th international conference on Business information systems. pp. 424-437. Springer-Verlag (2007)

12. Cruz, B.D., Jayaraman, B., Dwarakanath, A., McMillan, C.: Detecting Vague Words Phrases in Requirements Documents in a Multilingual Environment. In: 2017 IEEE 25th International Requirements Engineering Conference (RE). pp. 233-242 (2017). https://doi.org/10.1109/RE.2017.24

13. Cunningham, H.: Gate, a general architecture for text engineering. Computers and the Humanities 36(2), 223-254 (2002)

14. Eckhardt, J., Vogelsang, A., Femmer, H.: An approach for creating sentence patterns for quality requirements. In: Requirements Engineering Conference Workshops (REW), 24th IEEE International. pp. 308-315. IEEE (2016)

15. Elrakaiby, Y., Ferrari, A., Spoletini, P., Gnesi, S., Nuseibeh, B.: Using Argumentation to Explain Ambiguity in Requirements Elicitation Interviews. In: 2017 IEEE 25th International Requirements Engineering Conference (RE). pp. 51-60 (2017). https://doi.org/10.1109/RE.2017.27

16. Femmer, H., Vogelsang, A.: Requirements quality is quality in use. IEEE Software PP(99), 1-1 (2018). https://doi.org/10.1109/MS.2018.110161823 
17. Femmer, H.: Context-specific Requirements Engineering Quality

18. Femmer, H.: Reviewing Natural Language Requirements with Requirements Smells-A Research Proposal-. Proceedings of IDoESE (2013)

19. Femmer, H.: Automatic Requirements Reviews - Potentials, Limitations and Practical Tool Support. In: Felderer, M., Méndez Fernández, D., Turhan, B., Kalinowski, M., Sarro, F., Winkler, D. (eds.) Product-Focused Software Process Improvement. pp. 617-620. Springer International Publishing, Cham (2017)

20. Femmer, H., Fernández, D.M., Juergens, E., Klose, M., Zimmer, I., Zimmer, J.: Rapid requirements checks with requirements smells: two case studies. In: Proceedings of the 1st International Workshop on Rapid Continuous Software Engineering. pp. 10-19. ACM (2014)

21. Femmer, H., Fernández, D.M., Wagner, S., Eder, S.: Rapid quality assurance with requirements smells. Journal of Systems and Software 123, 190-213 (2017)

22. Femmer, H., Hauptmann, B., Eder, S., Moser, D.: Quality assurance of requirements artifacts in practice: A case study and a process proposal. In: International Conference on Product-Focused Software Process Improvement. pp. 506-516. Springer (2016)

23. Femmer, H., Mund, J., Fernández, D.M.: It's the activities, stupid! a new perspective on re quality. In: Requirements Engineering and Testing (RET), 2015 IEEE/ACM 2nd International Workshop on. pp. 13-19. IEEE (2015)

24. Femmer, H., Vogelsang, A.: Good RE Artifacts? I Know It When I Use It! Softwaretechnik-Trends: Vol. 37, No. 2 (2017)

25. Ferrari, A., Donati, B., Gnesi, S.: Detecting Domain-Specific Ambiguities: An NLP Approach Based on Wikipedia Crawling and Word Embeddings. In: 2017 IEEE 25th International Requirements Engineering Conference Workshops (REW). pp. 393-399 (2017). https://doi.org/10.1109/REW.2017.20

26. Ferrari, A., Spoletini, P., Donati, B., Zowghi, D., Gnesi, S.: Interview Review: Detecting Latent Ambiguities to Improve the Requirements Elicitation Process. In: 2017 IEEE 25th International Requirements Engineering Conference (RE). pp. 400-405 (2017). https://doi.org/10.1109/RE.2017.15

27. Ferrari, A.: Natural language requirements processing: from research to practice. In: Proceedings of the 40th International Conference on Software Engineering: Companion Proceeedings. pp. 536-537. ACM (2018)

28. Ferrari, A., Gori, G., Rosadini, B., Trotta, I., Bacherini, S., Fantechi, A., Gnesi, S.: Detecting requirements defects with nlp patterns: an industrial experience in the railway domain. Empirical Software Engineering pp. 1-50 (2018)

29. Ferrari, A., Spagnolo, G.O., Gnesi, S.: Pure: A dataset of public requirements documents. In: Requirements Engineering Conference (RE), 2017 IEEE 25th International. pp. 502-505. IEEE (2017)

30. Garcia, J., Popescu, D., Edwards, G., Medvidovic, N.: Toward a catalogue of architectural bad smells. In: Proceedings of the 5th International Conference on the Quality of Software Architectures: Architectures for Adaptive Software Systems. pp. 146-162. Springer-Verlag (2009)

31. Génova, G., Fuentes, J.M., Llorens, J., Hurtado, O., Moreno, V.: A framework to measure and improve the quality of textual requirements. Requirements engineering 18(1), 25-41 (2013)

32. Heck, P., Zaidman, A.: A systematic literature review on quality criteria for agile requirements specifications. Software Quality Journal 26(1), 127-160 (2018)

33. Hu, W., Carver, J.C., Anu, V., Walia, G.S., Bradshaw, G.L.: Using human error information for error prevention. Empirical Software Engineering pp. 1-33 (2018) 
34. Kasser, J.E., Tran, X.L., Matisons, S.P., et al.: Prototype educational tools for systems and software (pets) engineering. In: Engineering Education for a Sustainable Future: Proceedings of the 14th Annual Conference for Australasian Association for Engineering Education and 9th Australasian Women in Engineering Forum. p. 532. Australasian Association for Engineering Education (2003)

35. Kitchenham, B., Brereton, O.P., Budgen, D., Turner, M., Bailey, J., Linkman, S.: Systematic literature reviews in software engineering-a systematic literature review. Information and software technology 51(1), 7-15 (2009)

36. Lucassen, G., Dalpiaz, F., van der Werf, J.M.E., Brinkkemper, S.: Improving agile requirements: the quality user story framework and tool. Requirements Engineering 21(3), 383-403 (2016)

37. Mavin, A., Wilkinson, P., Harwood, A., Novak, M.: Easy approach to requirements syntax (ears). In: Requirements Engineering Conference, 2009. RE'09. 17th IEEE International. pp. 317-322. IEEE (2009)

38. Mund, J., Fernandez, D.M., Femmer, H., Eckhardt, J.: Does quality of requirements specifications matter? combined results of two empirical studies. In: Empirical Software Engineering and Measurement (ESEM), 2015 ACM/IEEE International Symposium on. pp. 1-10. IEEE (2015)

39. Pacheco, C., García, I., Reyes, M.: Requirements elicitation techniques: a systematic literature review based on the maturity of the techniques. IET Software (2018)

40. Pohl, K., Rupp, C.: Requirements engineering fundamentals, 1 sl ed. Rocky Nook (2011)

41. Rago, A., Marcos, C., Diaz-Pace, J.A.: Identifying duplicate functionality in textual use cases by aligning semantic actions (sosym abstract). In: Model Driven Engineering Languages and Systems (MODELS), 2015 ACM/IEEE 18th International Conference on. pp. 446-446. IEEE (2015)

42. Ramos, R., Castro, J., Araújo, J., Moreira, A., Alencar, F., Penteado, R.: Quality improvement for use case model. In: 2009 XXIII Brazilian Symposium on Software Engineering. pp. 187-195. IEEE (2009)

43. Rosadini, B., Ferrari, A., Gori, G., Fantechi, A., Gnesi, S., Trotta, I., Bacherini, S.: Using NLP to Detect Requirements Defects: An Industrial Experience in the Railway Domain. In: International Working Conference on Requirements Engineering: Foundation for Software Quality. pp. 344-360. Springer (2017)

44. Sabir, F., Rasool, G., Yousaf, M.: A lightweight approach for specification and detection of soap anti-patterns. INTERNATIONAL JOURNAL OF ADVANCED COMPUTER SCIENCE AND APPLICATIONS 8(5), 455-467 (2017)

45. SABRIYE, A.O.J., ZAINON, W.M.N.W.: An approach for detecting syntax and syntactic ambiguity in software requirement specification. Journal of Theoretical \& Applied Information Technology 96(8) (2018)

46. Sommerville, I.: Engenharia de software. PEARSON BRASIL (2011), https://books.google.com.br/books?id=H4u5ygAACAAJ

47. Unterkalmsteiner, M., Gorschek, T.: Requirements quality assurance in industry: why, what and how? In: International Working Conference on Requirements Engineering: Foundation for Software Quality. pp. 77-84. Springer (2017)

48. Vale, G., Figueiredo, E., Abílio, R., Costa, H.: Bad smells in software product line: A systematic review. Brazillian Conference on Software: Theory and Practice pp. 84-37 (2014)

49. Wagner, S., Fernández, D.M.: Analyzing text in software projects. In: The Art and Science of Analyzing Software Data, pp. 39-72. Elsevier (2016) 
50. Wilmink, M., Bockisch, C.: On the Ability of Lightweight Checks to Detect Ambiguity in Requirements Documentation. In: Grünbacher, P., Perini, A. (eds.) Requirements Engineering: Foundation for Software Quality. pp. 327-343. Springer International Publishing, Cham (2017)

51. Winkler, J., Vogelsang, A.: Towards Applicable Artificial Intelligence Tools in Requirements Engineering Using Visual Feedback (December) (2016). https://doi.org/10.13140/RG.2.2.16725.83680 